\title{
The influence of nicergoline on cerebral, systemic and cardiac hemodynamics in patients who have had an ischemic stroke
}

\author{
Victor V. Kuznetsov \\ State Institution “D. F. Chebotarev Institute of Gerontology NAMS of Ukraine”, Kyiv, Ukraine
}

Correspondence: kuznetsov66@ukr.net

Received: 16.11.2020; Accepted: 10.03.2021; Published: 26.04.2021

\begin{abstract}
The Aim of study was analysis of the effect of nicergoline on the cerebral, cardiac, systemic hemodynamics of patients after ischemic stroke, taking into account the hemispheric localization of the ischemic focus. Materials and methods. A comprehensive clinical and neurological examination was carried out in 38 elderly patients (mean age $64.3 \pm 2.5$ years) who had undergone atherothrombotic ischemic stroke in the carotid system (recovery period). There were 20 patients with left hemispheric stroke and 18 with right hemispheric stroke. All patients had arterial hypertension (AH). These were ACE inhibitors (enalapril 10-20 mg 1 tablet 2 times a day) and hydrochlorothiazide at a dose of $12.5 \mathrm{mg}$. Patients received nicergoline at a dose of $4 \mathrm{mg}$ IV drip for 10 days. A comprehensive examination was carried out before and after treatment with nicergoline. The Results of the study allowed us to conclude that the course of nicergoline intake in patients after ischemic atherothrombotic stroke improves the subjective state and reduces the severity of neurological disorders, cerebral hemodynamics, increases linear systolic blood flow velocities (LBFVsys) and reduces peripheral resistance in individual extra- and intracranial vessels of the carotid and vertebro-basilar basins. In patients with right and left hemispheric localization of stroke under the influence of nicergoline, LBFVsys increases in the right and left ICA, PCA, and VA. In addition, LBFVsys increases in patients with right hemispheric stroke in the right MBA; in patients with left hemisphere - in two MBA and BA. In ischemic stroke patients, nicergoline affects systemic and cardiac hemodynamics: it reduces the systemic and cardiac hemodynamics, decreases the systemic vascular resistance and increases the ejection fraction, changes the structure of the relationship between systemic and cerebral hemodynamics, forming a positive relationship between blood pressure and hemodynamics in the vessels of the vertebro-basilar basin. Thus, the complex positive effect of nicergoline on various levels of cerebral, systemic and cardiac hemodynamics in ischemic stroke patients gives grounds to recommend the inclusion of this drug in the rehabilitation system of this category of patients.
\end{abstract}

Key words: ischemic stroke; rehabilitation; nicergoline; hemispheric localization; hemodynamics

The high degree of disability in post-stroke patients (about $80 \%$ become disabled) determines the relevance of the development of effective methods of rehabilitation. The main tasks of the rehabilitation 
are: regeneration of the impaired functions, prevention of post-stroke complications, mental and social rehabilitation. The main principles of the rehabilitation are: early start of the rehabilitation activities, consistency and duration, stages, complexity and multidisciplinarity, active participation in the rehabilitation of the patient himself, his relatives and friends [1].

The regeneration of the impaired functions is based on the individual brain plasticity - the ability for compensation of the structural, functional and biochemical disorders [1]. Physiotherapy and medication methods of treatment are used to activate the processes of brain plasticity. A diversified approach to the rehabilitation of stroke patients determines the expediency of using drugs with a multimodal mechanism of action. One of these drugs is nicergoline (sermion) - a semi-synthetic derivative of the ergot alkaloid.

Nicergoline is a drug with a wide range of pharmacological activity. Nicergoline has a vasoactive, neurometabolic and neurotransmitter effect. In clinical and pharmacological plan, the primary effect of nicergoline is its selective vasodilatory effect, which is determined by the selective blockade of alpha-1 - adrenergic receptors in the vessel wall of the brain, which leads to the extension of the arteries, arterioles and capillary sphincters and, as a result, to a decrease in vascular resistance and an increase of the arterial blood flow [2 - 5].

The neurometabolic effects of nicergoline are only partly explained by its direct vasotropic action, that is, an improvement in hemodynamics and blood supply to the brain. In addition, nicergoline has: antihypoxic properties (activation of aerobic glycolysis processes, reduction of lactic acidosis, optimization of oxygen utilization); antioxidant effect (inhibition of the formation of free radicals and hydroperoxides); membrane stabilizing effect (normalization of calcium homeostasis in the nervous tissue) $[4,6]$.

The neurotransmitter properties of nicergoline are characterized by the effects on the cholinergic transmission. Under the influence of nicergoline, the synthesis of acetylcholine increases due to the activation of the choline acetyltransferase enzyme; the release of acetylcholine from presynaptic terminals is activated; decreases the breakdown of acetylcholine by inhibiting of the acetylcholinesterase enzyme; $[4,6,7]$.

In addition to cholinergic properties, nicergoline has a stimulating effect on adreno- and dopaminergic processes in the central nervous system by accelerating the turnover of norepinephrine and dopamine in the cortex and striatum [8,9]. Attention should also be focused on the ability of nicergoline to stimulate the reverse capture of glutamate and to prevent the development of glutamatemediated neurotoxic effects during hypoxia, to make an antiapoptotic effect, that is to inhibit the development of the phenomenon of excitotoxicity - the most important component of the "ischemic cascade" [4]. The antiapoptotic effect is an extremely valuable property of nicergoline as a neuroprotector. Recently, its influence on the processes of neuroplasticity [3 - 5] and the mechanisms of neuroprotection $[10,11]$ has been shown. These mechanisms are conditioned by the ability of nicergoline to act on endogenous neurotrophic factors. Experimental data indicate that old animals that received nicergoline have an increase in the concentration of nerve growth factor in the frontal parts of the brain [5]. Experimental studies have shown the neuroprotective effect of nicergoline during hypoxia, even in hypercapnia, when the cerebral vessels are in the state of dilatation, which indicates its direct effect on the brain parenchyma [3].

One of the mechanisms of the polymodal action is a disaggregating effect which is conditioned by a decrease in platelet aggregation and an increase in erythrocyte plasticity, which, in combination with the influence on the cerebral vessels, leads to the increase in regional cerebral blood flow in ischemic tissue $[2,5]$.

Currently, nicergoline is used in the treatment of patients with various forms of cerebrovascular diseases. In patients with the initial forms of cerebral atherosclerosis, nicergoline improves mnestic functions, potentiates the effect of antihypertensive drugs in patients with arterial hypertension $[10,12,13,14]$.

In patients who have had an ischemic stroke, nicergoline has a positive effect on the restoration of motor functions $[4,5]$. 
The wide range of pharmacotherapeutic activity and the polymodality of the mechanisms of action of nicergoline on the one hand and the hemispheric features of the action of some nootropic and neurotrophic drugs, on the other, determine the need to analyze the effect of nicergoline on cerebral hemodynamics, taking into account the hemispheric localization of the stroke.

Purpose of the work: a comprehensive analysis of the influence of nicergoline on cerebral, cardiac, systemic hemodynamics in patients who have had an ischemic stroke, taking into account the hemispheric localization of the ischemic focus.

\section{Materials and methods of the research.}

A comprehensive clinical and neurological examination was carried out for 38 elderly patients (average age $64.3 \pm 2.5$ years), who had come through atherothrombotic ischemic stroke in the carotid basin (recovery period). There are 20 patients with left hemispheric stroke, with right hemispheric -18 ones. All patients had arterial hypertension $(\mathrm{AH})$ and they took standard antihypertensive therapy in accordance with the European and Ukrainian recommendations for the treatment of arterial hypertension, consisting of ACE inhibitors (enalapril 10-20 mg 1 tab. 2 times per day) and hydrochlorothiazide at a dose of $12.5 \mathrm{mg}$.

Patients received nicergoline at a dose of $4 \mathrm{mg}$ by intravenous infusion for 10 days.

The examination program included: clinical and neurological examination, assessment of the level of daily activity according to the Bartel scale, clinical and neurological examination; ultrasound dopplerography of the vessels of the head and neck using the EN VISOR device (Philips) with the determination of LBFVsys and linear diastolic blood flow velocities (LBFVdias), vascular wall resistance index (Ri) and pulsatory index (Pi); transthoracic echocardiography using a Sonosite MicroMaxx device with a sector phased sensor (frequency 1.5-3.5 MHz).

MRI of the brain for the verification of the nature and localization of the ischemic focus on a 1.5 T Magnetom Vision Plus tomograph (SIEMENS);

The subjective state of the patients who suffered from an ischemic atherothrombotic stroke in the carotid basin was analyzed. Complaints presented by patients were assessed in points ("0" - no symptoms, " 1 " - minor symptoms, "2" - mild symptoms, "3" - severe symptoms).

A comprehensive examination was carried out before and after treatment with nicergoline.

\section{Results and discussion.}

An analysis of the subjective indicators of the state in patients with cerebral stroke was carried out. It was found that patients complained for headache $(94.7 \%)$, dizziness $(86.8 \%)$, noise in the head $(92.1 \%)$, unsteadiness when walking $(78.9 \%)$, decreased working capacity $(84.2 \%)$, emotional lability $(73.7 \%)$, memory impairment $(76.3 \%)$, sleep disturbance $(47.4 \%)$, a feeling of rush of blood to the head $(39.5 \%)$.

The severity of complaints of the patients with ischemic focus in the right and left hemisphere before and after treatment did not differ significantly. 
Table 1

Subjective complaints of stroke patients and their dynamics during treatment with nicergoline in points

\begin{tabular}{|l|l|l|l|l|}
\hline \multirow{2}{*}{ Complaints } & \multicolumn{3}{|c|}{ Ischemic stroke patients } \\
\cline { 2 - 5 } & \multicolumn{2}{|c|}{ On the right } & \multicolumn{1}{c|}{ On the left } \\
\cline { 2 - 5 } & $\begin{array}{c}\text { After } \\
\text { treatment } \\
\text { treatment }\end{array}$ & $\begin{array}{c}\text { After } \\
\text { treatment } \\
\text { treatment }\end{array}$ & $1.32 \pm 0.17^{*}$ \\
\hline Headache & $2.35 \pm 0.27$ & $1.32 \pm 0.17^{*}$ & $2.15 \pm 0.25$ & $1.45 \pm 0.09^{*}$ \\
\hline Dizziness & $1.82 \pm 0.19$ & $1.51 \pm 0.14$ & $1.74 \pm 0.07$ & $1.42 \pm 0.13^{*}$ \\
\hline Noise in the head, ears & $1.74 \pm 0.12$ & $1.31 \pm 0.08^{*}$ & $1.83 \pm 0.14$ & $1.29 \pm 0.13$ \\
\hline Emotional lability & $1 / 79 \pm 0.17$ & $1.48 \pm 0.19$ & $1.69 \pm 0.16$ & $1.02 \pm 0.17$ \\
\hline $\begin{array}{l}\text { Rush of blood to the } \\
\text { head }\end{array}$ & $1.32 \pm 0.15$ & $0.98 \pm 0.13$ & $1.29 \pm 0.16$ & $1.44 \pm 0.12^{*}$ \\
\hline $\begin{array}{l}\text { Unsteadiness when } \\
\text { walking }\end{array}$ & $1.67 \pm 0.14$ & $1.37 \pm 0.12^{*}$ & $1.87 \pm 0.14$ & \\
\hline Sleep disturbance & $1.12 \pm 0.21$ & $0.74 \pm 0.16$ & $1.25 \pm 0.19$ & $0.96 \pm 0.16$ \\
\hline Memory impairment & $1.72 \pm 0.24$ & $1.48 \pm 0.16$ & $1.87 \pm 0.22$ & $1.52 \pm 0.14$ \\
\hline
\end{tabular}

Note: ${ }^{*}-p<0.05$ statistically significant changes between indicators before and after treatment

According to the data presented in Tab. 1, the leading complaints in patients were headache, dizziness, unsteadiness when walking, noise in the ears and head. After a course of treatment with nicergoline, there was an improvement of the subjective state of the patients. In patients with left hemispheric stroke, the severity of all complaints decreased, but the decrease of the severity of headache, dizziness, noise in the head, and imbalance were statistically significant; in patients with right hemispheric stroke, the intensity of headache, dizziness and unsteadiness during walking significantly decreased.

In patients with ischemic stroke in neurological status, there were hemiparesis (100\%), impaired muscle tone $(76.3 \%)$, sensitivity $(68.4 \%)$, coordination $(65.7 \%)$, walking function and balance $(76.3 \%)$, subcortical reflexes $(89.5 \%)$, pathological foot reflexes $(68.4 \%)$, aphatic disorders $(13.1 \%)$, dysarthria (5.3\%), cognitive disorders (65.8\%).

Under the influence of the course treatment with nicergoline, no statistically significant changes in the degree of movement disorders were found. So, hemiparesis of the III-rd degree was established in $65.8 \%$ of patients before treatment, in $57.9 \%$ - after treatment; II-nd degree of hemiparesis in $34.2 \%$ and $42.1 \%$, respectively. Hemitype sensitivity disorders in $68.4 \%$ before treatment and in $65.7 \%$ after treatment, subcortical reflexes in $89.5 \%$ and $84.2 \%$, respectively. However, an improvement in the balance function should be noted, the interruption of which was leveled off in $10.5 \%$ of patients.

Thus, the course of nicergoline for the patients after ischemic atherothrombotic stroke has some positive effect on the clinical symptoms and improves certain indicators of neurological status. 
In the polymodal system of pharmacological action of nicergoline, its vasoactive and metabolic action occupies a special place. However, until now, no combined analysis of the effect of nicergoline on cerebral, systemic and cardiac hemodynamics and, in particular, in stroke patients has been carried out. Concerning this, we analyzed the effect of nicergoline on cerebral blood flow in patients with stroke during the recovery period. It was found that under the influence of a course of nicergoline, cerebral blood circulation was improved due to an increase in LBFVsys and a decrease in peripheral resistance in individual vessels of the carotid and vertebro-basilar basins. However, the hemodynamic mosaic of these changes has hemispheric features (Tab. 2).

In patients with left hemispheric stroke, LBFVsys statistically significantly increased in the extra- and intracranial vessels of the carotid (ICA, MBA) and vertebro-basilar (VA, BA, PCA) basins of the affected and intact hemispheres. The maximum increase of LBFVsys is observed in the MBA of both hemispheres.

In patients with stroke localization in the right hemisphere under the influence of nicergoline, LBFVsys statistically significantly increased in both ICA and VA, PCA and in the right MBA.

Table 2

Dynamics of LBFVsys in ischemic stroke patients before and after nicergoline treatment

\begin{tabular}{|c|c|c|c|c|}
\hline \multirow{3}{*}{ Vessels } & \multicolumn{4}{|c|}{ LBFVsys indices in stroke patients } \\
\hline & \multicolumn{2}{|c|}{ In the right hemisphere } & \multicolumn{2}{|c|}{ In the left hemisphere } \\
\hline & Before treatment & After treatment & Before treatment & After treatment \\
\hline CCA (I) & $60.75 \pm 4.54$ & $56.42 \pm 3.41$ & $59.35 \pm 2.44$ & $62.06 \pm 1.93$ \\
\hline CCA (A) & $75.79 \pm 4.40$ & $71.22 \pm 2.95$ & $64.56 \pm 2.30$ & $67.80 \pm 2.74$ \\
\hline ICA (I) & $57.66 \pm 2.60$ & $66.22 \pm 2.88^{*}$ & $53.26 \pm 1.83$ & $63.43 \pm 2.48^{*}$ \\
\hline ICA (A) & $60.73 \pm 2.60$ & $71.70 \pm 2.89^{*}$ & $56.69 \pm 2.91$ & $65.12 \pm 2.45^{*}$ \\
\hline VA (I) & $33.03 \pm 1.39$ & $38.52 \pm 1.75^{*}$ & $35.79 \pm 1.77$ & $41.88 \pm 1.87^{*}$ \\
\hline VA (A) & $37.73 \pm 1.85$ & $43.21 \pm 1.74^{*}$ & $35.80 \pm 1.79$ & $41.47 \pm 1.57^{*}$ \\
\hline MBA (I) & $86.08 \pm 4.66$ & $95.35 \pm 5.25$ & $89.64 \pm 3.65$ & $105.84 \pm 3.78^{*}$ \\
\hline MBA (A) & $81.87 \pm 3.76$ & $93.17 \pm 2.62^{*}$ & $76.91 \pm 4.76$ & $89.91 \pm 4.60^{*}$ \\
\hline PCA (I) & $47.32 \pm 1.77$ & $54.80 \pm 2.34^{*}$ & $45.75 \pm 1.82$ & $53.63 \pm 1.87^{*}$ \\
\hline PCA (A) & $45.09 \pm 1.45$ & $53.60 \pm 1.52^{*}$ & $45.27 \pm 1.68$ & $52.74 \pm 1.64^{*}$ \\
\hline BA & $52.01 \pm 3.76$ & $57.25 \pm 2.85$ & $51.69 \pm 3.48$ & $61.38 \pm 3.57^{*}$ \\
\hline
\end{tabular}

Note: ${ }^{*}-p<0.05$ statistically significant changes between indicators before and after treatment. I - intact hemisphere, A - affected hemisphere. 
In patients who have had an ischemic stroke in the left hemisphere, nicergoline statistically significantly reduces peripheral resistance ( $\mathrm{Pi}$ ) in the left CCA (from 1,83 $\pm 0,10$ to $1,58 \pm 0,07$ after) and left PCA $(0,79 \pm 0,02,0,73 \pm 0,01$ after respectively).

In patients who have had a right hemispheric stroke, nicergoline reduces peripheral resistance on the affected side in the CCA (from 2,12 $\pm 0,12$ to 1,77 $\pm 0,10$ ), in intact VA (from 1,16 $\pm 0,04$ to $1,03 \pm$ 0,05 ) and PCA (from $0,76 \pm 0,02$ to $0,70 \pm 0,01$ ) and in both MBA (from $0,91 \pm 0,05$ to $0,81 \pm 0,04$ on the right side and from $0,86 \pm 0,02$ to $0,82 \pm 0,03$ on the left side).

Table 3

Dynamics of the pulsation index $(\mathrm{Pi})$ in ischemic stroke patients before and after treatment with nicergoline

\begin{tabular}{|c|c|c|c|c|}
\hline \multirow{3}{*}{$\begin{array}{l}\text { Vessels of the } \\
\text { carotid and } \\
\text { vertebro-basilar } \\
\text { basins }\end{array}$} & \multicolumn{4}{|c|}{ Pulsation index (Pi) in patients who have had a stroke } \\
\hline & \multicolumn{2}{|c|}{ In the right hemisphere } & \multicolumn{2}{|c|}{ In the left hemisphere } \\
\hline & Before treatment & After treatment & Before treatment & After treatment \\
\hline CCA I & $2.12 \pm 0.12$ & $1.77 \pm 0.10^{*}$ & $1.62 \pm 0.07$ & $1.56 \pm 0.07$ \\
\hline CCA A & $1.54 \pm 0.08$ & $1.55 \pm 0.08$ & $1.83 \pm 0.10$ & $1.58 \pm 0.07^{*}$ \\
\hline ICA I & $1.09 \pm 0.04$ & $1.00 \pm 0.04$ & $1.08 \pm 0.06$ & $1.01 \pm 0.04$ \\
\hline ICA A & $0.93 \pm 0.04$ & $0.92 \pm 0.04$ & $1.04 \pm 0.04$ & $0.99 \pm 0.04$ \\
\hline VA I & $1.23 \pm 0.07$ & $1.20 \pm 0.07$ & $1.16 \pm 0.05$ & $1.13 \pm 0.05$ \\
\hline VA A & $1.16 \pm 0.04$ & $1.03 \pm 0.05^{*}$ & $1.17 \pm 0.09$ & $1.25 \pm 0.07$ \\
\hline MBA I & $0.91 \pm 0.05$ & $0.81 \pm 0.04^{*}$ & $0.83 \pm 0.02$ & $0.78 \pm 0.03$ \\
\hline MBA A & $0.86 \pm 0.02$ & $0.82 \pm 0.03^{*}$ & $0.81 \pm 0.05$ & $0.78 \pm 0.04$ \\
\hline PCA I & $0.79 \pm 0.03$ & $0.76 \pm 0.02$ & $0.77 \pm 0.03$ & $0.73 \pm 0.03$ \\
\hline PCA A & $0.76 \pm 0.02$ & $0.70 \pm 0.01^{*}$ & $0.79 \pm 0.02$ & $0.73 \pm 0.01^{*}$ \\
\hline BA & $0.85 \pm 0.05$ & $0.84 \pm 0.04$ & $0.82 \pm 0.04$ & $0.82 \pm 0.04$ \\
\hline
\end{tabular}

Note: ${ }^{*}$ - $p<0.05$ statistically significant changes between indicators before and after treatment I - intact hemisphere, A - affected hemisphere 
Thus, the course use of nicergoline in patients after ischemic stroke improves cerebral hemodynamics, that is evidenced by an increase in LBFVsys, a decrease in peripheral resistance indices in the vessels of the carotid and vertebro-basilar basin. In patients with ischemic stroke localized in the left hemisphere, an increase in LBFVsys is observed in the extra- and intracranial vessels of the affected (ICA, VA, MBA, PCA) and intact IBCA, VA, MBA, PCA) hemispheres and in the BA; in patients with right hemispheric stroke, predominantly in the extracranial (ICA и VA) vessels of both hemispheres and in the intracranial vessels in the BA, left MBA, and both PCA.

It should be emphasized that in elderly patients, an ischemic stroke appears, as a rule, against the background of concomitant pathology of the cardiovascular system (arterial hypertension, stenocardia, various forms of heart rhythm disturbances, heart failure). Violations of the contractile ability of the heart, arrhythmias facilitate the strengthening of the cerebrovascular insufficiency $[15,16]$. At the same time, the changes in cerebral hemodynamics and the phenomenon of cerebral hypoxia lead to a violation of the central mechanisms of regulation of cardiovascular activity [17]. In particular, cardiac arrhythmias lead to disorders of cerebral blood flow and the formation of pathogenetic mechanisms of cardioembolic stroke, and interruption in the functioning of suprasegmental vegetative structures make worse cardiac disorders [18, 19].

The presence of concomitant pathology of the cardiovascular system in elderly patients who have had a stroke, and the peculiarities of the pharmacokinetics of drugs of this category of patients (agerelated characteristics of metabolism and drug interaction) determine the expediency of conducting a comprehensive research of the effect of nicergoline on both cerebral hemodynamics and parameters of the systemic and intracardiac hemodynamics. It should be noted that the available data about the effect of nicergoline on systemic and cerebral hemodynamics are contradictory: so, in the study of Gara (1993), the effect of nicergoline on systemic and cerebral hemodynamics was studied. As a result, it was found that nicergoline reduces the minute volume of blood (MVB), cardiac index $(\mathrm{CI})$ and increases total peripheral vascular resistance (TPVR) [20]. Other researchers have noted an increase in MVB and a decrease in TPVR under the influence of nicergoline in patients with ischemic stroke. Perhaps these contradictions are due to the fact that in the study of Gar (1993) the group of patients with ischemic stroke was heterogeneous, because the study group included patients both in the acute and in the rehabilitation period and the age range was from 25 to 80 years [20]. In the study by Kuznetsova (1999), there were only elderly patients and in the recovery period of stroke.

Analysis of changes in the structural and functional state of the heart in patients who had an ischemic stroke under the influence of nicergoline showed the presence of a statistically significant change in the parameters of systemic vascular resistance, MVB and ejection fraction (EF). In patients, nicergoline causes an increase in EF, MVB against the background of a decrease in TPVR (Tab. 4). It should be noted that the above hemodynamic changes occurred against the background of a statistically significant decrease in systolic blood pressure (SBP) (systolic blood pressure).

Table 4

Dynamics of indicators of systemic and cardiac hemodynamics in ischemic stroke patients before and after treatment with nicergoline.

\begin{tabular}{|l|l|l|}
\hline \multicolumn{1}{|c|}{ Indicators } & \multicolumn{1}{|c|}{ Before treatment } & Affter treatment \\
\hline Heart rate beats / min & $70.56 \pm 0.71$ & $70.58 \pm 0.74$ \\
\hline Systolic blood pressure (mm Hg) & $130.63 \pm 1.05$ & $126.69 \pm 1.00^{*}$ \\
\hline Diastolic blood pressure (mm Hg) & $82.19 \pm 0.88$ & $81.88 \pm 0.86$ \\
\hline Average blood pressure (mm Hg) & $98.40 \pm 0.93$ & $97.82 \pm 0.84$ \\
\hline
\end{tabular}




\begin{tabular}{|c|c|c|}
\hline Body area, $\left(\mathrm{m}^{2}\right)$ & $1,88 \pm 0,04$ & $1,87 \pm 0,04$ \\
\hline Aorta, $(\mathrm{cm})$ & $3.26 \pm 0.60$ & $3.24 \pm 0.68$ \\
\hline Left atrium, $\mathrm{cm}$ & $3.47 \pm 2.31$ & $3.47 \pm 2.31$ \\
\hline Wall thickness of the right ventricular, $\mathrm{cm}$ & $0.46 \pm 0.01$ & $0.47 \pm 0.01$ \\
\hline End-diastolic size of the right ventricular, $\mathrm{cm}$ & $2.55 \pm 0.11$ & $2.61 \pm 0.11$ \\
\hline End-diastolic size of the left ventricular, $\mathrm{cm}$ & $4.89 \pm 0.12$ & $4.91 \pm 0.12$ \\
\hline End-systolic size of the left ventricular, $\mathrm{cm}$ & $3.25 \pm 0.10$ & $3.13 \pm 0.11$ \\
\hline Shortening fraction $(\%)$ & $33.75 \pm 0.73$ & $36.17 \pm 0.80^{*}$ \\
\hline The thickness of the interventricular septum, $(\mathrm{cm})$ & $1.32 \pm 0.04$ & $1.32 \pm 0.04$ \\
\hline The posterior wall of the left ventricular, $\mathrm{ml}$ & $1.17 \pm 0.03$ & $1.17 \pm 0.03$ \\
\hline End-systolic volume (ESV), $\mathrm{ml}$ & $43.96 \pm 3.69$ & $40.13 \pm 3.94$ \\
\hline ESV index $\mathrm{ml} / \mathrm{m}^{2}$ & $23.48 \pm 1.88$ & $20.21 \pm 2.54$ \\
\hline End-diastolic volume (EDV) $\mathrm{ml}$ & $113.65 \pm 7.18$ & $115.22 \pm 7.29$ \\
\hline Index (EDV) $\mathrm{ml} / \mathrm{m}^{2}$ & $61.03 \pm 3.81$ & $59.76 \pm 4.69$ \\
\hline Stroke volume $\mathrm{ml}$ & $70.85 \pm 3.72$ & $74.87 \pm 3.62$ \\
\hline Stroke volume index $\mathrm{ml} / \mathrm{m}^{2}$ & $38.12 \pm 2.06$ & $40.40 \pm 1.98$ \\
\hline Total peripheral resistance & $1729.47 \pm 75.11$ & $1543.19 \pm 71.99^{*}$ \\
\hline Cardiac index $\mathrm{ml} / \mathrm{m}^{2}$ & $2687.79 \pm 149.00$ & $2859.96 \pm 143.35$ \\
\hline Minute blood volume, $\mathrm{ml}$ & $4993.31 \pm 264.88$ & $5301.04 \pm 261.06$ \\
\hline Ejection fraction $(\%)$ & $62.64 \pm .01$ & $65.87 \pm 1.05^{*}$ \\
\hline Left-Ventricular Myocardial Mass (LVMM) & $197.67 \pm 9.60$ & $191.27 \pm 10.43$ \\
\hline Left-Ventricular Myocardial Mass Index (LVMMI) & $112.28 \pm 6.38$ & $112.28 \pm 6.38$ \\
\hline Left-Ventricular Relative Wall Thickness Index & $52.41 \pm 1.77$ & $52.41 \pm 1.77$ \\
\hline
\end{tabular}

As is known, in cerebral stroke, the mechanisms of autoregulation of cerebral homeostasis are disrupted and the effect of systemic hemodynamics on cerebral circulation increases [9, 10]. Taking into account the effect of nicergoline on cerebral blood flow and central hemodynamics, the analysis of the interconnections of indicators of cerebral blood flow (LBFVsys, Pi) with cardiac and systemic hemodynamics in stroke patients before and after taking a course of nicergoline was carried out. It was found that in patients who have had an ischemic stroke, before taking nicergoline, there were noted statistically significant paradoxical negative interconnections between SBP and LBFVsys in the right ICA $(\mathrm{r}=-0.58)$ and in both PCA $(\mathrm{r}=-0.70$ and $\mathrm{r}=0.61$, respectively). After a course of nicergoline treatment, the correlation structure is characterized by the formation of positive interconnections between SBP and 
LBFVsys in the left PCA $(r=0.56)$ and BA $(r=0.68)$, which, to a certain extent, indicates about the reorganization of the interconnections between systemic and cerebral hemodynamics in vertebro-basilar basin in patients who have had an ischemic stroke. The complex positive effect of nicergoline on various levels of cerebral, systemic and cardiac hemodynamics in patients who have had an ischemic stroke gives reasons to recommend the inclusion of this drug in the rehabilitation system of this category of patients.

\section{Conclusions:}

1. Course taking of nicergoline in patients who have had an ischemic atherothrombotic stroke improves the subjective state and reduces the severity of neurological disorders.

2. In patients who have had an ischemic stroke, nicergoline improves cerebral hemodynamics, increases LBFVsys and decreases peripheral resistance in separate extra- and intracranial vessels of the carotid and vertebro-basilar basins.

3. In patients with right and left hemispheric localization of stroke under the influence of nicergoline, LBFVsys increases in the right and left ICA, PCA and VA. In addition, LBFVsys increases in patients with right hemispheric stroke in the right MBA; in patients with left hemispheric stroke - in both MBA and BA.

4. The taking of course of nicergoline by the patients who have had an ischemic stroke affects systemic and cardiac hemodynamics: it reduces the systemic vascular resistance and increases the ejection fraction.

5. In patients who have had an ischemic stroke, nicergoline changes the structure of the interconnections between systemic and cerebral hemodynamics, forming a positive interconnections between BP and hemodynamics in the vessels of the vertebro-basilar basin.

6. The complex positive effect of nicergoline on the various levels of cerebral, systemic and cardiac hemodynamics in patients who have had an ischemic stroke gives reasons to recommend the inclusion of this drug in the rehabilitation system of this category of patients.

Information about Author:

Viktor V. Kuznetsov - DSc (Medicine), Leading Researcher of Department of Vascular Pathology of the Brain, Department of Rehabilitation of Neurological Patients; https://orcid.org/0000-0002-2044-6507; Web of Science Researcher ID W9357-2019 


\section{References}

1. Kadykov, A.S.; Chernikova, L.A.; Shakhparonova, N.V. Rehabilitation of neurological patients (in Russian). Medpress-inform: Mocow, 2009, p 560

2. Windish, M. Drugs that enhance cognitive functions (nootropics) (in Russian). EBEVE: Mocow, 2001, p 23. doi: 10.3233/JAD-121729

3. Cattabeni, F. Protein kinase $C$ in synaptic plasticity: A molecular target in the treatment of cognitive disorders. Dement Geriat Cogn Dis 1997, 8,1, pp. 6-11

4. Winblad, B.; Fioravanti, M.; Dolezal, T.; Logina, I.; Gospodinov, I. et al. Therapeutic use of nicergoline. Clin Drug Invest 2008, 28, pp. 533-552. doi:10.2165/00044011-200828090-00001

5. Zylberman, M.R.; Fontana, M.; Dolce, G. Effects of nicergoline on learning, mood and behaviour in hemiplegic patients undergoing rehabilitation. Ergot alkaloids and aging brain: an update on nicergoline. Ed. Kugler, J.; Agnoli, A. Excerpta Medica: Amsterdam, 1988, pp. 57-63

6. Loganovsky, K.N.; Yuriev, K.L. Pharmacological properties and therapeutic potential of Sermion. Neuropsychiatric effects of Sermion (in Russian). Ukr. Med. Magazine 2005, 2, 46, pp. 59-75.

7. Ogawa, N.; Asanuma, M.; Hirata, H.; Kondo, Y.; Kawada, Y. et al. Cholinergic deficits in aged rat brain are corrected with nicergoline. Arch Gerontol Geriatr 1993, 16, pp. 103-110.

8. Calza, L.; Giardino, L. Neuroprotection: a realistic goal for aged brain? Adv Exp Med Biol 2004,541, pp. 153-168. doi: 10.1007/978-1-4419-8969-7_9

9. Moretti, A.; Carfagna, N.; Caccia, C.; Carpentieri, M. et al. Effect of ergolines on neurotransmitter systems in the rat brain. Arch Int Pharmacodyn Ther 1988, 294, pp. 33-45.

10. Odinak, M.M.; Emelin, A.Yu.; Lobzin, V.Yu. Cognitive impairment in cerebrovascular pathology (in Russian). VMedA: St. Petersburg, 2006, p 158

11. Caraci, F.; Chisari, M.; Frasca, G.; Canonico, P.L.; Battaglia, A. et al. Nicergoline, a drug used for age-dependent cognitive impairment, protects cultured neurons against $\beta$-amyloid toxicity. Brain Res 2005, 1047, pp. 30-37. doi: 10.1016/j.brainres.2005.04.004

12. Rudenko, G.M.; Muzychenko, A.P. Results of a clinical study of the drug Sermion (analysis of data from the pharmacological committee) (in Russian). Moscow, 1987, p 31.

13. Яхно Н.Н., Захаров В.В., Локшина А.Б. и др. Деменции. Руководство для врачей. 2-е изд. М: МЕДпресс-информ 2010; 272. Yakhno, N.N.; Zakharov, V.V.; Lokshina, A.B. et al. Dementia. A guide for doctors. 2nd ed. (in Russian). MEDpress-inform: Moscow, 2010, p 272

14. Crook, T.H. Nicergoline: Parallel evolution of clinical trial methodology and drug development in dementia. Dement Geriat Cogn Dis 1997, 8, 1, pp. 22-26. doi: 10.1159/000106667

15. Suslina, Z.A. Essays on angioneurology (in Russian). Atmosphere: Moscow, 2005, pp. 108-121

16. Pyshkina, L.I.; Fedin, A.I. The use of vasoactive drugs for occlusive lesions of the main arteries of the head

(in Russian). Abstracts of the VII All-Russian Congress of Neurologists: N. Novgorod,1995, p. 287

17. Sorokina, T.A.; Shafro, I.S.; Krievane, D.A. The state of cerebral circulation in older patients with coronary artery disease (in Russian). Ischemic heart and brain disease, mental illness in geriatric practice (abstracts): Irkutsk, 1990, pp. 115-117

18. Ena, L.M.; Egorova, M.S. Features of Holter ECG monitoring in elderly and senile patients with cardioembolic stroke (in Russian). Archives of Clinical and Experimental Medicine 2009, 2, pp. 227-230 19. Spasennikov, B.A. Dyscirculatory encephalopathy (pathogenetic, clinical and pharmacological aspects). Abstract of the dissertation (in Russian). RSMU: Moscow 1996, p.278

20. Kuznetsova S.M., Slivak E.A., Prikhodko V.Yu. (1999) The effect of serion on cerebral hemodynamics in elderly patients with residual symptoms of ischemic acute cerebrovascular accident in the carotid system (in Russian). Ukr. Med. Magazine 1999, 5,13, pp. 18-21 